\title{
Interventions to improve health literacy among Aboriginal and Torres Strait Islander Peoples: a systematic review
}

Simone Nash ${ }^{1}$ and Amit Arora ${ }^{1,2,3,4^{*}}$ (D)

\begin{abstract}
Background: Aboriginal and Torres Strait Islander peoples continue to experience poorer health outcomes than other population groups. While data specific to Indigenous Australians are scarce, a known social health literacy gradient exists linking low health literacy and poor health outcomes within many minority populations. Improving health literacy among Indigenous Australians is an important way to support self-determination and autonomy in both individuals and communities, by enhancing knowledge and improving health outcomes. This review aims to rigorously examine the effectiveness of health literacy interventions targeting Aboriginal and Torres Strait Islander peoples.
\end{abstract}

Methods: A systematic review across six databases (The Cochrane Library, PubMed, Embase, SCOPUS, ProQuest Dissertation and Thesis and Web of Science) was performed for publications evaluating interventions to improve health literacy among Indigenous Australian adults using search terms identifying a range of related outcomes.

Results: Of 824 articles retrieved, a total of five studies met the eligibility criteria and were included in this review. The included studies evaluated the implementation of workshops, structured exercise classes and the provision of discounted fruit and vegetables to improve nutrition, modify risk factors for chronic diseases, and improve oral health literacy. All interventions reported statistically significant improvement in at least one measured outcome. However, there was limited involvement of the Aboriginal and Torres Strait Islander community members in the research process and participant retention rates were sub-optimal.

Conclusion: There is limited evidence on interventions to improve health literacy in Indigenous Australian adults. Participation in interventions was often suboptimal and loss to follow-up was high. Future studies co-designed with Aboriginal and Torres Strait Islander community members are needed to improve health literacy in this population.

Keywords: Aboriginal, Torres Strait Island, Indigenous, First Nations Peoples, Health literacy, Australia, Social determinants of health, Socio-economic inequality, Systematic review

\footnotetext{
* Correspondence: a.arora@westernsydney.edu.au

${ }^{1}$ School of Health Sciences, Western Sydney University, Locked Bag 1797, Penrith, NSW 2751, Australia

${ }^{2}$ Translational Health Research Institute, Western Sydney University, Locked

Bag 1797, Penrith, NSW 2751, Australia

Full list of author information is available at the end of the article
}

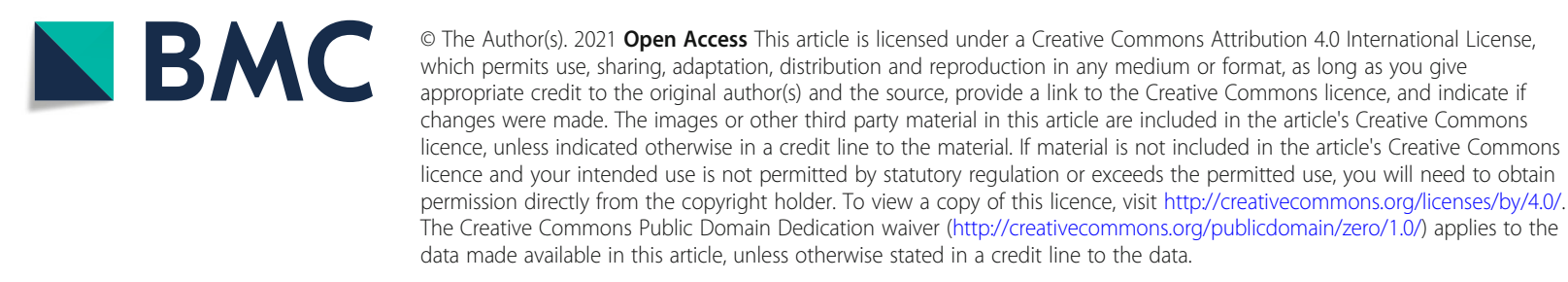




\section{Background}

Health literacy is a social construct which can be defined as the skill required to perform tasks such as reading, writing, understanding, and interpreting the basic health information and services in order to make appropriate health decisions [1]. The term encompasses not only a range of cognitive skills which allow people to access and utilise health information to enhance their ability to engage in promotion and maintenance of good health [1,2]; but also the skills required for the incorporation of of health information, navigation, processing, problem-solving and decision-making [3]. Health literacy, a term first proposed in the 1970's, is a concept that continues to evolve and be redefined. The Calgary Charter on Health Literacy was formulated by the World Health Organization and defines the term as the ability of both the public and healthcare professionals to locate, comprehend, share and utilise health information [4]. A recent systematic review investigating the meaning of health literacy defined it as the ability of an individual to obtain and translate knowledge and information in order to maintain and improve health in a way that is appropriate to the individual and system contexts [5]. Health literacy encompasses all the skills which contribute to the ability to live a healthful life [3]. In this respect, health literacy is recognised as an important factor that not only involves the patient/ consumers' and the individuals/organisations involved in the provision of care [6]; but is also a concept that pertains to broader societal health practice and the ways in which health information is sought and shared among population groups (distributed health literacy) $[7,8]$.

Health literacy is a determinant of health [9], and is conceptualised on three hierarchal levels: functional, communicative/interactive and critical; beginning with basic skills through to the most complex which include the ability to critically analyse health information and affect change on the health of self and others [10]. Health literacy is critical to health promotion and encompasses the environmental, political and social factors which either impede or improve health outcomes [11]. A social gradient related to health literacy has been identified in all national health surveys [9] linking low levels of health literacy levels with poorer health outcomes among minority population groups [12].

The understanding of the concepts of health literacy gains deeper meaning in the context of culture. Cultural health beliefs affect how people think and feel about their health, when and from whom they seek health care, and how they respond to prevention and management of health conditions. Definitions of health literacy that do not recognise the potential effects of culture on the communication and understanding of health information neglect deeper interpretations of what it means to be health literate [10]. This is especially important given the ethnic and linguistic diversity in Australia with the urgent need to improve cultural literacy of service providers [13].

Aboriginal and Torres Strait Islander peoples are the First Peoples of Australia and comprise about $3.3 \%$ of the Australian population [14, 15]. Many groups of Aboriginal and Torres Strait Islander peoples do not receive the same opportunity to achieve and maintain physical and mental health as nonIndigenous Australians [16]. The overall burden of disease in Indigenous populations remains more than twice that of their non-Indigenous counterparts, with prevalence of chronic diseases including mental illnesses, respiratory, renal and cardiovascular diseases of particular concern [17]. Life expectancy for many Aboriginal and Torres Strait Islander Australians remains around 17 years lower than that of other Australians [18], with a recent report showing no improvement in these statistics in the last decade [19]. The economic cost of the burden of disease is substantial, and while almost $50 \%$ more per capita is spent on Indigenous health, [12, 20] spending falls well short for the significantly more complex needs of this population [20]. Though progress has been made in recent years to improve the health and well-being of Aboriginal and Torres Strait Islander peoples [19], advancements in health outcomes within the general population has meant that the gap between the health of Indigenous and non-Indigenous Australians continues to widen $[19,21]$.

The reasons for continued Indigenous health disparities are numerous and complex and begin with the profound and enduring impact of colonisation and subsequent dilution of language, culture, disconnection from country, systemic discrimination and mistreatment [22]. The holistic understanding of health and wellbeing for Indigenous Australians involves the whole community throughout the entire life-course and includes broad issues such as social justice, equity, and rights, as well as traditional knowledge, traditional healing, and connection to Country [23]. A number of theories have been proposed to demonstrate relationships between health literacy, health outcomes and Indigeneity [24]. To date, these theories lack the support of data, though understanding the potential relationships that may be at play is an important strategy in determining where intervention might be effective [24]. The key components of the health literacy relationship pathways outlined by Australian Commission on Safety and Quality in Health Care [24] include: how individuals' access and utilise health services; the interactions that occur between consumers 
and providers; and how people manage and exert control over their own health [24]. These health literacy pathways are reflected in the outcome measures used in this review (knowledge or skills; attitudes, motivation or behaviour changes; self-efficacy; self-management; engagement with and use of available health care services; health status/outcomes).

Australian State and Territory Health Services continue to attempt to address the issues impacting these pathways and the social determinants of Indigenous health, including the continuing need for accessible and culturally appropriate health services for Aboriginal and Torres Strait Islander peoples. In 2007, The Council of Australian Governments (COAG) identified Closing the Gap as a national priority, and though progress has been made in some domains, many require more work and unfortunately the status of others have worsened [25]. The recently published Closing the Gap Report 2020 highlights the struggle to meet these targets continues to this day [26]. The reasons for the shortcomings of this initiative are many, and include unrealistic targets, inadequate Indigenous involvement and other issues of mismanagement [18]. It is worth noting that while the social determinants of Indigenous health remain unchanged, reaching such targets will remain unlikely [18].

Understanding health literacy and its relationship to the status of Indigenous health is an important step in working toward promoting health literacy in Aboriginal and Torres Strait Islander peoples [27]. The 2006 Australian Bureau of Statistics (ABS) survey [28] and the 2018 Australian National Health Survey [29] did not report on health literacy data related of Aboriginal and Torres Strait Islander peoples. However, it is thought to be very likely that health literacy levels among Aboriginal and Torres Strait Islander peoples are lower than among other non-Indigenous population groups [24]. In recent years, several systematic reviews have been conducted both in Australia and internationally examining interventions for individuals with low health literacy [16, 30, 31], with most of these targeting specific sub-groups. Other systematic reviews have examined the interventions to improve health literacy among Indigenous people affected by cancer [32] or evaluated health literacy interventions among minority populations more broadly [31, 33-35]. To date, research investigating Aboriginal and Torres Strait Islander peoples and limited health literacy levels is sparse, and more is required in order to increase awareness of the issue in order to increase information, access and resources. To the best of our knowledge this is the first systematic review to evaluate the effectiveness of health literacy interventions targeting Indigenous Australians and aims to add knowledge through the synthesis of available evidence related to interventions to improve health literacy among Indigenous Australian adults.

\section{Methods}

This review was conducted based on the Preferred Reporting Items for Systematic Reviews and Metaanalysis (PRISMA) guidelines [36]. The protocol for this review is published and registered with The PROSPERO International Register of Systematic Reviews (PROSPERO 2020: CRD42020130529) [37].

\section{Eligibility criteria}

The inclusion and exclusion criteria were formulated based on the Population, Intervention, Comparator, Outcome and Study Design (PICOS) framework [38] (Table 1).

Table 1 Eligibility for inclusion in the systematic review

\begin{tabular}{|c|c|}
\hline Inclusion Category & Inclusion Criteria \\
\hline Population & $\begin{array}{l}\text { - Identify as Aboriginal and/or Torres Strait Islander people } \\
->18 \text { years old }\end{array}$ \\
\hline Intervention & $\begin{array}{l}\text { Includes improvement of a health outcome of interest: } \\
\text { - Health literacy } \\
\text { - Knowledge or skills } \\
\text { - Attitudes/Motivation } \\
\text { - Behaviour change } \\
\text { - Self-efficacy } \\
\text { - Self-management } \\
\text { - Engagement with/Use of services } \\
\text { - Health status }\end{array}$ \\
\hline Study Design & $\begin{array}{l}\text { Experimental designs } \\
\text { - Randomised Control Trials } \\
\text { - Quasi-randomised Control Trials } \\
\text { - Matched Comparison Groups } \\
\text { - Controlled Before and After } \\
\text { - Pre/Post } \\
\text { - Interrupted Time Series }\end{array}$ \\
\hline Publication & $\begin{array}{l}\text { English only } \\
\text { Reports quantitative measurements }\end{array}$ \\
\hline
\end{tabular}




\section{Types of participants}

To be considered for inclusion, studies must have been conducted with adult Indigenous Australian participants over 18 years of age. In studies recruiting Indigenous Australians and participants of other backgrounds, data specific to Indigenous Australians was sought from the author/s. Where no such data were obtainable such studies were excluded. No restriction was made regarding the gender of participants.

\section{Types of interventions}

Any intervention that the authors reported to be aimed at improving health literacy were included. This may include interventions that aimed to change any predefined aspect of health literacy in an individual or population, including:

- Knowledge about risk factors, disease, prevention and/or treatments

- Attitudes, confidence or beliefs

- Ability to care for self (including disease selfmanagement), self-efficacy and autonomy

- Health-related literacy skills

- Help-seeking behaviours, awareness, access to or utilisation of health care services

- Engagement with and cultural safety of health care services and health professionals

\section{Types of comparators}

Comparator/control groups included non-intervention or usual care group, alternative interventions, matched samples receiving the same intervention and historical control groups.

\section{Types of outcome measures}

Studies that described an outcome measure related to health literacy were included in this review. These outcome measures may have included:

- Validated health literacy measurement tool

- Knowledge or skills

- Attitudes, motivation or behaviour changes

- Self-efficacy

- Self-management

- Engagement with and use of available health care services

- Health status/outcomes

\section{Types of studies}

All studies describing an intervention with one of the following designs were included in this review: Randomised control trials (RCTs), quasi randomised control trials, matched comparison group designs, controlled before and after studies, pre-post-test studies or interrupted time series. Qualitative studies and conference proceedings were excluded from this review. In cases where more than one paper was published by the authors detailing the same study, relevant data from all papers were extracted as one study.

\section{Information sources}

The search began in consultation with a professional Health Sciences librarian, who assisted in guiding the authors to relevant databases. The following databases were subsequently searched: Cochrane Central Register of Controlled Trials (CENTRAL) (The Cochrane Library), MEDLINE (OVID) and Embase (OVID). Additionally, Proquest Dissertation and Thesis, SCOPUS and Web of Science were searched for eligible grey literature. Furthermore, a parallel manual search of the reference lists of all eligible studies and previously published systematic reviews on health literacy was performed. No restriction was made on the date of publication. The initial search was concluded on the 24th April 2019, was reperformed on the 14th October 2019 and subsequently updated on the 20th June 2020. Studies were restricted to English language only.

\section{Search strategy}

The Population Intervention Comparator Outcome and Study Design (PICOS) framework [38] was used to devise the search terms. A combination of Medical Subject Headings $(\mathrm{MeSH})$ terms and keywords were formulated in consultation with a professional Health Sciences Librarian and peer-reviewed to ensure completeness. These terms included all identified variations of "Australian Aboriginal peoples," "Indigenous Australians" and "Torres Strait Islander people" combined with all identified headings and suggested terms for "health literacy," "health education," "access to information," "consumer health information," "patient education," "health knowledge, attitudes and practice," "self-care," "self-concept" and "self-efficacy."

Combinations of keywords and terms using Boolean operators, truncation, phrase searching, and Medical Subject Heading (MeSH) were used in the search strategies. The initial search string was developed and tested using Embase (OVID) (see search strategy Additional file 2). This search was subsequently adapted to the syntax and subject headings of the other databases employed.

\section{Study selection}

Studies identified through database searches, grey literature, theses and manual searches were subsequently exported to EndNote X9 [39] for removal of duplicates, screening and selection. Two reviewers ( $\mathrm{SN}$ and $\mathrm{AA}$ ) independently screened the articles based on the eligibility 
criteria. Abstracts of the studies that were considered to potentially meet the criteria for this review were read by two reviewers (SN and AA). Full texts of studies considered to be eligible were then read by two reviewers ( $\mathrm{SN}$ and AA). Study authors were contacted to seek additional information in case of any uncertainty on eligibility. A total of two attempts were made to contact the study authors, and if no response was received studies were screened for eligibility based on the information available. Tabulated details of the studies that were read in full and subsequently excluded have their reason/s for exclusion reported in Additional file 3. The study selection process was carried out with reference to the Preferred Reporting Items for Systematic Reviews and Meta-Analysis (PRIS MA) checklist [36] (Additional file 1).

\section{Data collection process}

A standardised data extraction form was developed and pilot-tested based on a checklist presented in The Cochrane Handbook for Systematic Reviews of Interventions [40]. Data were extracted for one study initially to ensure consistency across reviewers and ensure all relevant data were captured. Data from all the included studies were extracted independently by two reviewers (SN and AA). The data extracted from each included study included information on the first author, year of publication, information on the participants/sample, follow-up period, particulars of the intervention/s, study setting, design, funding, data analysis techniques, as well as a brief summary of outcome measures, results and conclusion for each study. For studies where necessary data were missing, the corresponding author/s were contacted with a maximum of two attempts to seek clarification. Where no response was received, data extraction was completed using the information available. The completed data summary table is included here (Table 2).

\section{Assessment of methodological quality}

The methodological quality of each study was assessed independently by two reviewers (SN and AA) using the appropriate standardised critical appraisal tool produced by the Joanna Briggs Institute (JBI) [47].

\section{Data synthesis}

Following the tabulation of extracted data, a narrative was created to provide descriptive synthesis of the included studies. Outcomes were described from the data using differences in means, proportions, risk ratios with relevant 95\% confidence intervals.

\section{Results}

\section{Results of the search}

Initial searches retrieved 824 citations from all databases and manual searches. A total of 791 studies remained following removal of 33 duplicates. A total of 768 studies were excluded as they did not meet the inclusion criteria for this review during initial screening. The remaining $(n=23)$ studies were read in full by two authors (SN and AA). A total of 18 studies were excluded after reading full-text and their reason/s for exclusion were tabulated (Additonal file 3). Finally, five studies were found to meet the criteria for this systematic review and were thereafter included in data synthesis. A meta-analysis was not conducted due to the small number of included studies and heterogenous interventions and outcomes [40]. A PRISMA flow diagram was constructed, detailing the identification, screening and eligibility process (Fig. 1).

\section{Study characteristics}

Five studies met the inclusion criteria and were included in this review. These included one stepwedged randomised trial with longitudinal sub-study [41, 42], one pragmatic randomised trial [43], one randomised controlled trial [44], and two pre-post longitudinal quasi-experimental studies $[45,46]$. The included studies were published between 2012 and 2018 and were written in English language. The studies took place in a range of locations with widely varied demographic characteristics including: communities in the very remote regions of the Northern Territory [41, 42]; urban and regional areas of Western Australia [46]; as well as metropolitan and rural areas of South Australia [43, 44] and south east Queensland [45]. Three studies reported consultation and collaboration with the communities/populations of interest during the development of projects, and the employment of Indigenous staff in their implementation [42, 44, 45]. Loss to follow-up and poor attendance were a significant issue for several studies $(n=3)[41,43,45]$. This was despite organisers offering increased flexibility and fiscal incentives aimed at increasing participation [41, 44].

The exact number of participants included in these studies was indeterminable due to the Brimblecombe et al. [42] study offering community-wide interventions. The authors of this study estimate the population of the communities in which the intervention was offered to be approximately 8515 persons, $95 \%$ of whom identify as Indigenous [42]. The total definitive number of Indigenous participants across all other studies was 902 . This number included both men and women, though the majority of participants involved in all studies were female ( $n=686$ or $76.05 \%)$. Participants ranged in age from 18 to $74+$ years. Two studies incentivised participation, with participants offered gifts, vouchers, or prizes for attendance at workshops/demonstrations or for the completion of questionnaires [41, 44]. 


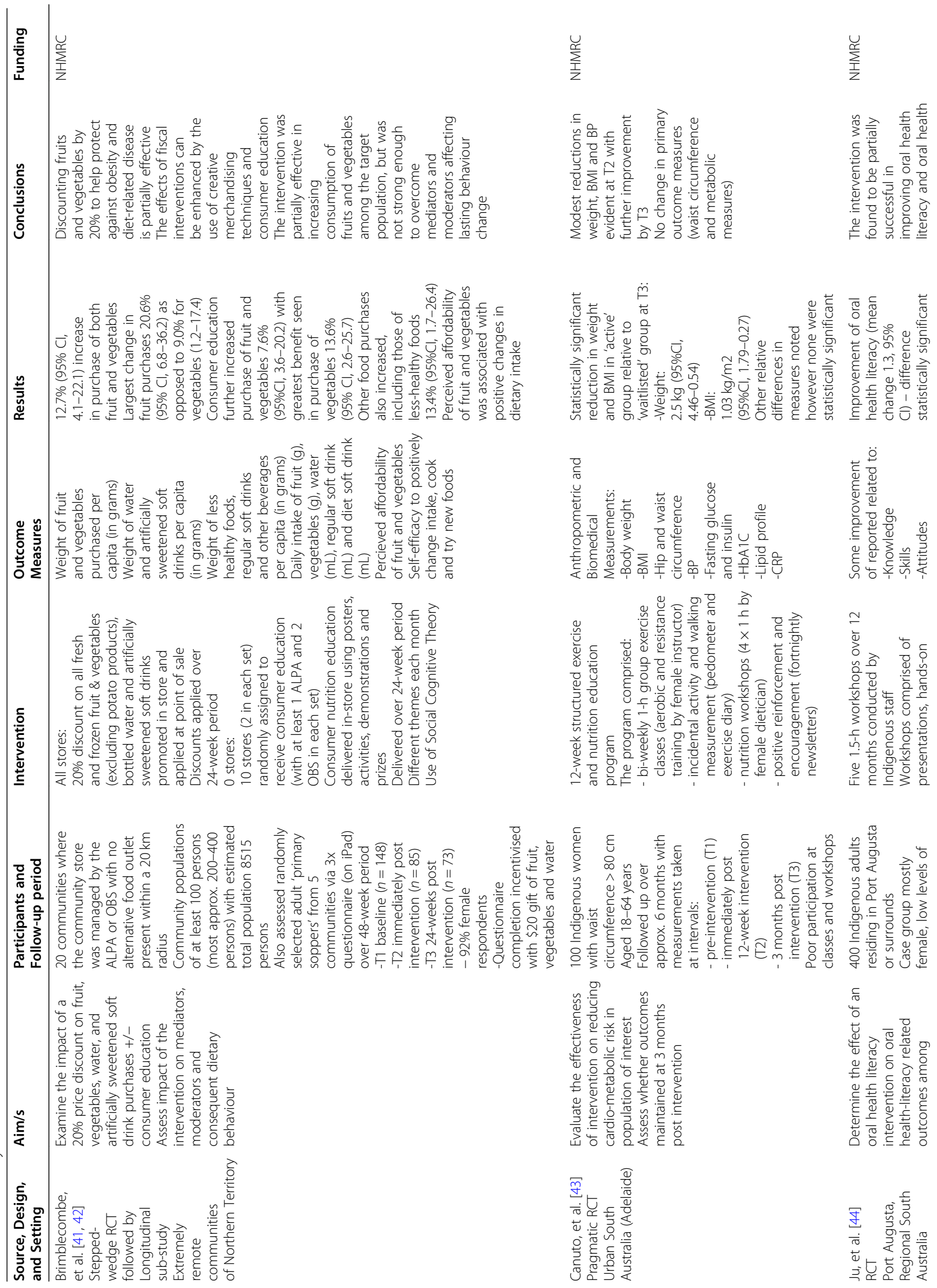




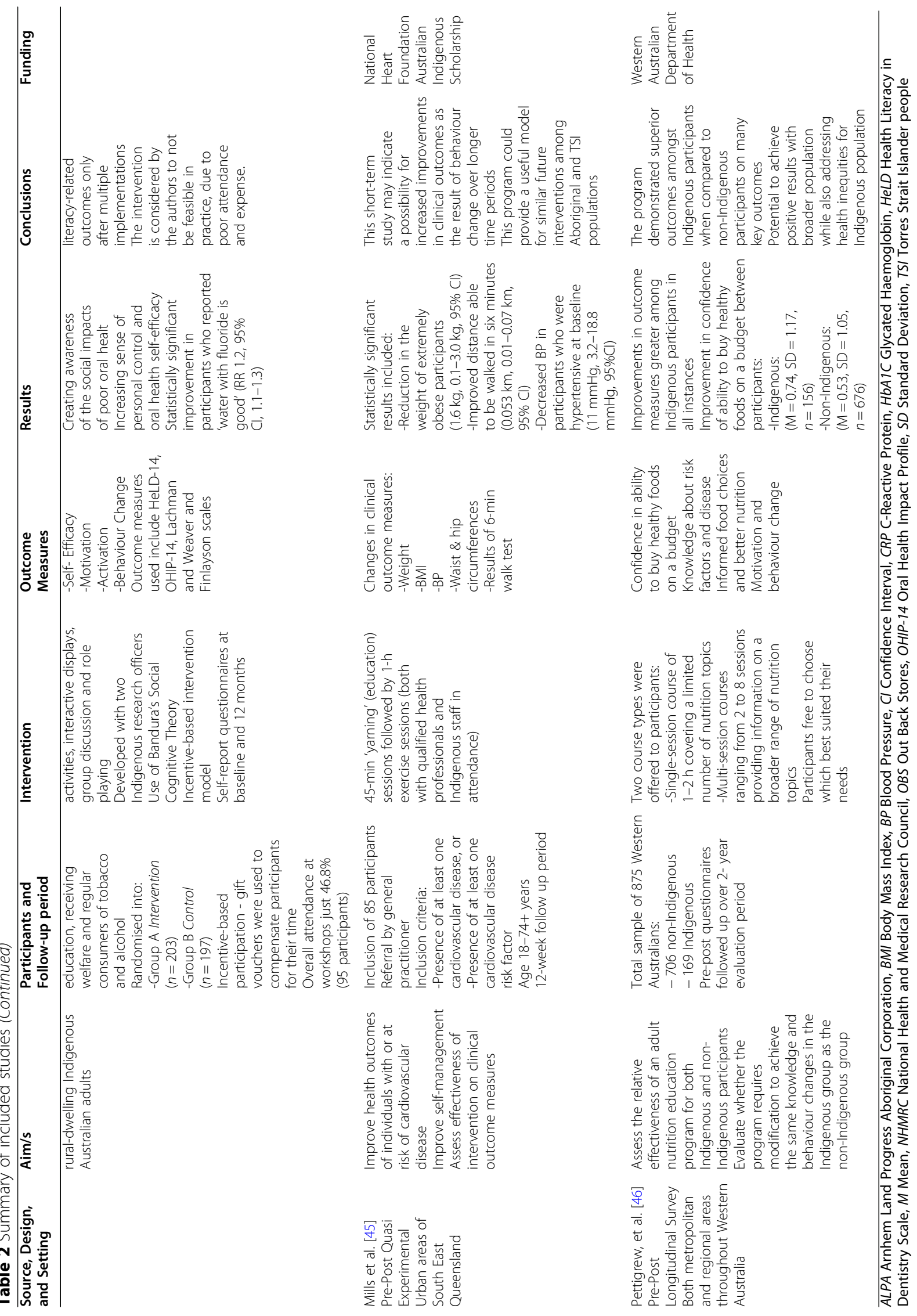




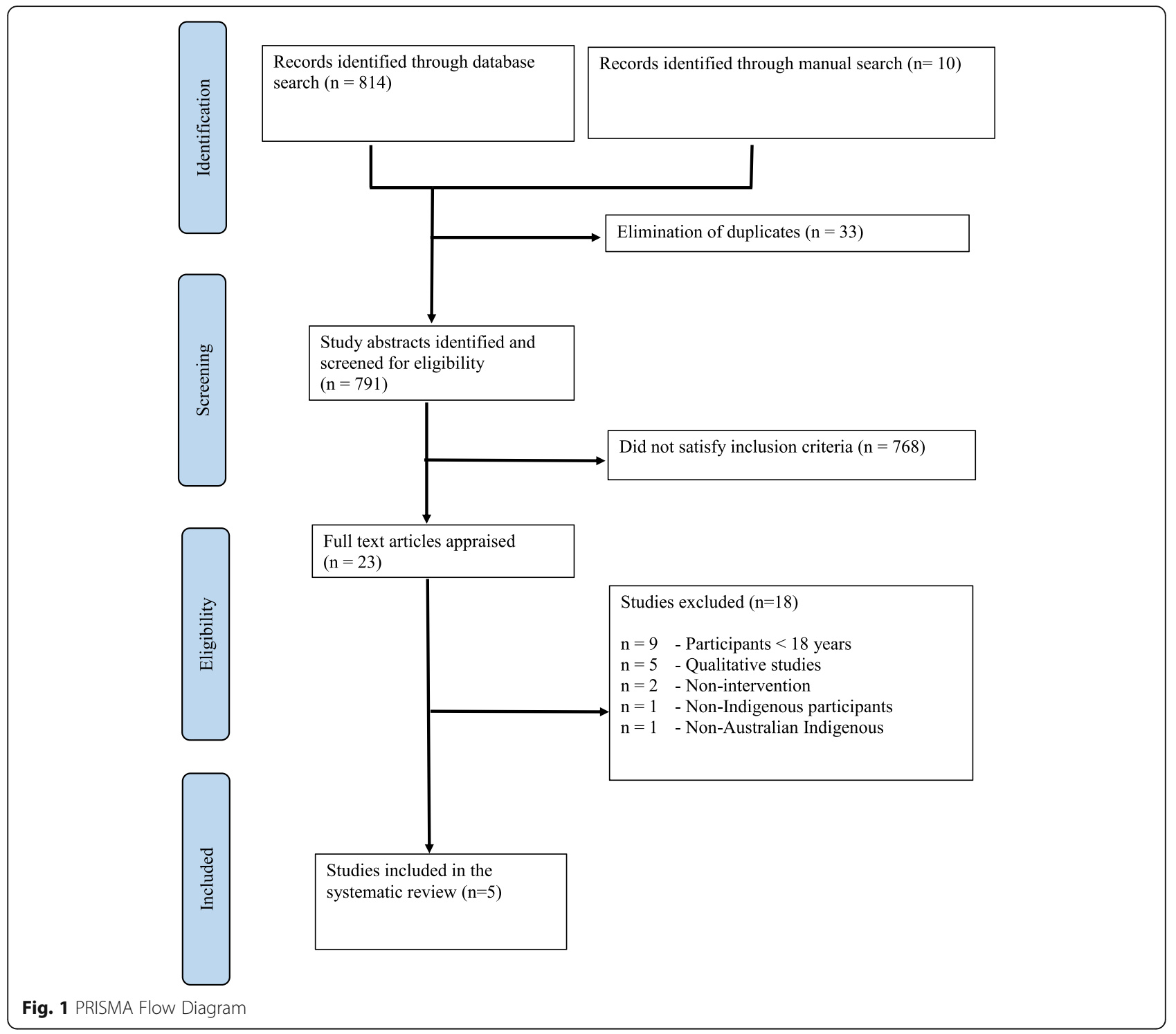

\section{Intervention strategies and their effectiveness}

There were two main types of intervention strategies among the studies: workshops/group education sessions [43-46] and reduction in the pricing of fruit and vegetables [42].

\section{Workshops and group education}

All of the studies included in this review provided some form of education to participants in order to improve their health literacy. The workshops or group education sessions included structured exercise classes [43, 45], nutrition/cooking workshops [43, 46], discussions/roleplaying, presentations and other related learning activities [44].

Pettigrew et al. [46] recorded greater improvements in the self-reported confidence of Indigenous participants in their ability to buy healthy foods on a budget following attendance at workshop/s when compared to non-Indigenous participants $\quad($ Mean $=0.74, \quad \mathrm{SD}=1.17$ among Indigenous participants and Mean $=0.53, \mathrm{SD}=$ 1.05 among non-Indigenous participants). This occurred across all outcome measures with no modification of the intervention for cultural specificity.

The study conducted by Canuto et al. [43] also offered education on nutrition, though these workshops focussed on nutrition in relation to reducing the risk of cardio-metabolic disease specifically. The results of this education were assessed using anthropometric and biomedical measures. Attendance at a structured exercise and nutrition education program over a period of 12-weeks for Indigenous women with waist circumferences $>80 \mathrm{~cm}$ was found to contribute to a reduction in weight from baseline (Mean: $2.5 \mathrm{~kg}$, 95\% CI, 4.46-0.54) and BMI (Mean: 
$1.03 \mathrm{~kg} / \mathrm{m} 2,95 \% \mathrm{CI}, 1.79-0.27$ ) [41]. Differences in other anthropometric measurements and biomedical markers were statistically insignificant, though this may be due to the relatively short follow up period of only 6-months.

On a similar theme, Mills and colleagues [45] also aimed to address risk of chronic cardiovascular disease in at-risk individuals through the offering of exercise groups and 'yarning' sessions (a form of circular dialogue used to build relationships and share information in Indigenous Australian cultures [48]). Like the Canuto et al. [43] study, outcomes were assessed by calculation of changes in biophysical measures, though this time over a very brief follow up period of just 12-weeks. Participation in the program described in this study was demonstrated to lead to a reduction in the weight of extremely obese participants $(1.6 \mathrm{~kg}, 0.1-3.0 \mathrm{~kg}, 95 \% \mathrm{CI})$, improved distance able to be walked in six minutes $(0.053 \mathrm{~km}$, $0.01-0.07 \mathrm{~km}, 95 \% \mathrm{CI}$ ) as well as decreased blood pressure (BP) in participants who were hypertensive at baseline $(11 \mathrm{mmHg}, 3.2-18.8 \mathrm{mmHg}, 95 \% \mathrm{CI})$ [45]. While the aim of these studies may have been to improve health literacy levels, it is possible that such results may have been influenced by other factors.

In the study conducted by Ju and colleagues [44], participation in workshops was demonstrated to improve overall oral health literacy (Mean change $=1.3,1.1-1.6$, 95\% CI) [44]. The number of participants recognising the benefits of fluoridated drinking water saw the most significant elevation (94.0 intervention vs. 76.8 control, $\mathrm{RR}=1.2,95 \% \mathrm{CI})$. Other parameters saw improvements, though these were neither statistically significant nor repeated under all scenarios used in the study [44].

\section{Reduction in the cost of fresh/frozen produce and low- sugar beverages}

Brimblecombe et al. [42] demonstrated that the introduction of an in-store $20 \%$ discount on fruit, vegetable, water and artificially sweetened soft drinks was associated with a positive effect on sales of fruit and vegetables (12.7\% increase in weight of fruit and vegetables purchased, 4.1-22.1, 95\% CI). This effect was enhanced with the addition of consumer education at point-of-sale (increasing sales by a further 7.6\%, 3.6-20.2, 95\% CI) [42]. The perceived affordability of fresh produce was later also positively associated with healthy food choices and dietary changes in the population of interest [41].

\section{Outcomes}

The results of all included studies demonstrate statistically significant improvement in at least one health literacy related outcome measure following participation in the intervention. However, each study assessed the effect of the intervention/s using different measures or tools with only one study using a measurement tool to assess health literacy [44].

One randomised controlled trial [44] utilised the specially formulated 'Health Literacy in Dentistry' scale to quantify changes in the primary outcome (health literacy related to oral health). This tool was specifically developed in collaboration with the population of interest for this study to ensure cultural safety and sensitivity [44]. Secondary outcomes of this intervention were also measured using scale to ascertain awareness of the social impacts of oral health, sense of personal control to maintain good oral health, oral health-related selfefficacy and general dental knowledge [44].

Both of the studies examining interventions to mitigate risk or impact of cardiovascular disease in at risk or affected individuals $[43,45]$, did so through implementation of structured group exercise classes, and measured outcomes through the collection biomedical and anthropometric data from participants.

Three studies offered some form of nutrition education as part of their broader interventions [42, 43, 46]. Outcome measures for each of these studies were however quite different; with Brimblecombe [42] measuring changes in purchases of fruits, vegetables and beverages; Canuto et al. [43] and Mills et al. [45] assessing outcomes using measurements of biophysical markers and anthropometry; and Pettigrew et al. [46] utilising selfreported improvements in knowledge and confidence to measure post-intervention outcomes. Using these varied measures, each study successfully generated quantifiable results from the intervention/s employed.

\section{Assessment of methodological quality}

Three studies were assessed using the checklist for Randomised Control Trials [42-44] and two using the checklist for Pre-Post Quasi-Experimental studies [45, 46] produced by the JBI [47]. The results of the application of these quality assessment instruments have been reported descriptively here and can be found in tabulated version in Additional file 4 and 5. All eligible studies were included in this review irrespective of their methodological quality.

Each included study demonstrated partially adequate methodological quality. Whilst all five scored strongly when assessed using the JBI checklists, issues with participation in interventions and/or retention of participants in several [41, 43, 44]; as well as a lack of consultation with the population of interest and missed opportunities for co-design and collaboration in others $[43,46]$ detracted from otherwise generally robust methodological quality.

The randomised control trials [42-44] demonstrated adequate randomisation; blinding, treatment, outcome measurement; and suitable statistical analysis. One pre- 
post longitudinal study [46] also proved to be of partially adequate methodological quality, although it (and the study by Ju et al. [44]) relied solely on self-reported data for comparison of pre- and post-intervention, and therefore required participants to possess adequate literacy levels to complete questionnaires. The Pettigrew et al. [46] study also lacked post-intervention measurement of biophysical data which may have demonstrated actual behaviour change, though weakness was identified by the authors and may have been beyond the scope of the study.

The pre-post quasi-experimental study conducted by Mills and colleagues [45] was of strong methodological quality and measured biophysical markers at baseline and predetermined intervals throughout the study period to assess outcomes. This quality of this study was also bolstered by prioritised building of trust, promotion of community control throughout the designing and implementation of the intervention and through the involvement of Indigenous researchers.

The Canuto et al. [43] and Ju et al. [44] studies reported very poor attendance (with only around $40 \%$ attendance at workshops/classes for each), and high loss to follow-up. This substantially limited sample sizes, effectiveness of programs, and the amount of data collected. Ju et al. [44] utilised multiple imputation to compensate for missing data while the Canuto et al. [43] study reported only on available data. There was also some concern of possible sample contamination voiced by the authors of the oral health literacy trial, [44] as the pilot of the study had been held in close geographic proximity to the trial itself.

\section{Discussion}

The purpose of this systematic review was to identify, appraise, and create a synthesis of interventions to improve health literacy amongst Indigenous Australian adults. Of the five studies that were included in this review, four included interventions to improve health literacy in relation to lifestyle factors and nutrition; and one addressed oral health literacy. Studies varied in size and scope from the involvement of thousands of individuals across multiple communities, to clusters of small groups with as few as one hundred participants involved in others. Interventions were commonly provided in the form of workshops or classes. Each included study was somewhat successful in fulfilling its objectives with statistically significant improvement in at least one outcome related to health literacy measured.

In order to increase the scope of this review and capture a broad range of interventions targeting all possible measures of health literacy, this review did not require the outcomes to be assessed using a specific health literacy measurement tool such as The Rapid Assessment of
Health Literacy in Medicine (REALM), The Test of Functional Health Literacy in Adults (TOFHLA) or The Newest Vital Sign [49]. As none of these instruments have been found to comprehensively assess an individual's true capacity [50] and have been criticised by some for over-simplifying health literacy [49], this review included all manner of outcome measures related to the improvement of knowledge, skills, attitudes, motivation or behaviour changes, self-efficacy or improved selfmanagement, engagement with and use of available health care services as well as health outcomes; which may all be considered the result of enhanced health literacy. While changes in measurements of these outcomes may be impacted by other factors, they have been included for completeness as they are considered to be important aspects of health literacy able to be influenced by the application of intervention [5].

The interventions included in this review comprised oral health literacy workshops [44]; as well as the delivery of cooking, nutrition and exercise classes [43, 45, 46]. Attendance at education sessions such as these provides participants with the opportunity to gain knowledge; and may also enhance motivation through shared experiences and increased sense of social connection. Workshops have been successfully employed as a means to provide targeted interventions to improve health literacy among varied groups; including those with certain disease pathologies or risk factors, senior citizens, refugees, parents of paediatric patients, as well as among other Indigenous populations (New Zealand Māori, Native American, Taiwanese Aboriginals) [51-56].

The study by Brimblecombe and colleagues [42] was the largest included in this review, and measured the impact of a discount on fruit, vegetables, water and lowsugar beverages in twenty communities across very remote regions of Western Australia. This intervention increased health literacy in the target population by providing education, increasing awareness and facilitating an increase in the sale of healthier foods. The intervention led to an increase in the amount of fruits and vegetables purchased from stores during the period of the intervention and this affect appeared to persist following its completion. A modest added impact was noted with the addition of in-store education (aimed at improving health literacy related nutrition) which was randomly assigned to half of the communities involved in the study. These results are consistent with those of similar studies conducted amongst the general population in both New Zealand and the Netherlands [57, 58]. Other Australian research has also shown positive health benefits could possibly be derived from the imposition of revenue-neutral taxes on less healthy foods to subsidise fresh fruit and vegetables [59]. A similar strategy is already used in countries around the world, where a 
'sugar-tax' on soft drinks has been implemented with the aim of combating obesity and related noncommunicable diseases [60]. This strategy has demonstrated effectiveness by increasing public awareness about sugar consumption, decreasing purchases of sugar sweetened beverages (SSBs) and encouraging SSB manufacturers to decrease the sugar content of their products [60]. Pressure to implement such a tax in Australia is currently mounting [61], though any such measure must of course be applicable to the entire population and not target specific sub-groups.

Two studies included in this review [41, 44] utilised some form of incentive in order to encourage participation or reward/retain participants. Incentives are seen by many as a means to improve health outcomes [62], recognise the valuable contributions of individuals to the determination of robust research outcomes, and enhance recruitment and retainment in research [63]. Incentives offered to participants included vouchers, 'prizes,' fruit and vegetable baskets and intervention-related paraphernalia (such as water bottles, toothbrushes, dental mirrors etc.). The use of incentives in research and health promotion is commonly seen in successful studies involving Indigenous participants [64-66] and is soundly grounded in behaviour modification theory [67]. However, incentivising research remains a somewhat contentious strategy with some arguing that it may undermine autonomous decision-making by being coercive; compromise the integrity of results by inadvertent overrecruitment of those from lower-socioeconomic backgrounds; or that it may even reduce altruism and intrinsic motivation, with the possibility of becoming counterproductive and leading to decreased participation [63]. It is impossible to determine whether these factors were at play in the poor participation and significant loss to follow-up experienced in the studies included in this review.

Four of the five included studies reported some form of community consultation during development [4245].. The studies utilising a participatory approach also employed Indigenous research officers, workshop facilitators and/or educators. Recently, other studies embracing continual collaboration and participant co-design have been successfully working to develop interventions to address health literacy among hospitalised patients [68] and to support new Aboriginal and Torres Strait Islander parents who have experienced complex trauma [69]. In the studies included here, this community involvement did not always translate into engagement and participation; with poor attendance at interventions and loss to follow-up reported even where investment in Indigenous consultation and involvement was considerable. This is particularly noted in the oral health literacy intervention conducted by Ju and colleagues [44].
The methodological quality of the included studies was partially adequate, with deficits found to have predominantly occurred due to missed opportunities for involvement of the population of interest during the research process, poor participation in interventions and significant loss to follow-up. The various randomised controlled trials [42-44] achieved strong results in terms of achievement of adequate randomisation, concealment of treatment groups and blinding. The pre-post studies $[45,46]$ also scored well on the standardised checklist employed [47]. Methods for measurement of outcomes across all studies were reliable, although Canuto et al. [43] reported some minor difficulties with the use of equipment to measure $\mathrm{BP}$ and waist circumference given the differing shapes of the participants involved in their study. Also, the use of self-reported questionnaires to measure outcomes $[41,44,46]$ may have been problematic in that they assume a level of literacy and English proficiency sufficient to comprehend and appropriately respond to questions, which some participants may not have possessed. The Brimblecombe et al. [41] and Pettigrew et al. [46] studies also lacked any collection of biophysical data which may have been demonstrative of actual behaviour change post-intervention, though this was beyond the scope of these studies.

The results of this review highlight the profound complexities of addressing the health gap and the effects of existing interventions to improve health literacy among Indigenous Australians on addressing health inequities remain unknown. This can be seen to demonstrate the importance of ameliorating Indigenous selfdetermination through continual consultation and collaboration with community members from project inception to completion. In the wake of centuries of systemic devaluing of Indigenous Australian culture, the promotion of positive representatives and role models is critical to the development of a healthier, strength-based Indigenous identity [22].

It is important to consider also that all Aboriginals and Torres Strait Islander peoples themselves belong to smaller population groups, with over 500 distinct nations spread across the continent, each with its own world views, beliefs, traditions and many with distinct dialects/language [70]. None of the included studies report delivering interventions in the local dialects of participants, or report being responsive to the heterogeneity of Aboriginal and Torres Strait Islander peoples' culture, though translators were offered to participants of the Pettigrew et al. study [46]. Differences in world view and language barriers between Aboriginal and Torres Strait Islander peoples and service providers are known to impede health literacy [71]. The use of First language could serve to empower communities, promote autonomy and create shared understandings between health care 
professionals and Indigenous communities that may otherwise be lost in translation [72]. Only one of the studies included in this review reports consideration of between or within-group diversity [45]. This may mean that the other interventions used could plausibly have been more or less effective when applied to different Indigenous nations [16], a factor that might have been mitigated by increasing collaboration and partnership with communities during the conception, design and implementation of research protocols.

\section{Strengths and limitations}

This is the first systematic review to evaluate interventions to improve health literacy among Indigenous Australians. While the search encompassed six databases, including both peer-reviewed studies and grey literature and was supplemented by manual reference list searches, it is possible that some relevant studies may have been missed. This review was strengthened by the range of terms used to describe health literacy outcomes in the search of databases, leading to the capture of a broader variety of studies. However, this approach may have also limited the review by contributing to the heterogeneity of outcome measures seen among included studies, which hampered comparisons between interventions and made pooling of the results statistically to undertake a meta-analysis impossible. The use of these broad search terms may also have compromised the results of this review in that outcomes could have plausibly been influenced by other factors and not have been the result of increased health literacy. As with all systematic reviews, the results and conclusions depend on the quality of the published literature, with small sample sizes making it impossible to determine whether null findings represented a true lack of effect or simply reflected limitations in statistical power. The use of scales to measure health literacy has been criticised as being overly simplified and may limit the scope of studies if used in isolation $[3,46]$. Whilst this review includes studies using health literacy scales, it also considers outcome measures related to the improvement of knowledge, skills, attitudes, motivation or behaviour changes, self-efficacy or improved self-management, engagement with and use of available health care services as well as health status outcomes to be indicative of a change in health literacy.

\section{Implications}

To the best of our knowledge, this is the first systematic review that aims to synthesise the evidence on interventions to improve health literacy in Aboriginal and Torres Strait Islander populations. However, many opportunities remain for important future research. Future health literacy studies may benefit from an investigation of reasons for poor retention rates of Aboriginal and Torres Strait Island participants in research, and from an exploration of ways to modify approaches which may lead to improved participation throughout the research process. These may include encouraging the use of codesign and participatory approaches, use of language and culturally appropriate communication, the incorporation of community-specific perceptions of health and wellbeing in interventions and/or increasing engagement with Indigenous staff. Currently, researchers in public health use "adequate" and "inadequate" or "low" levels of health literacy which may have different meanings across different settings. The public health field will benefit if researchers clearly specify relevant cut-points of distinguishing different levels of health literacy. There may also be value in the creation of standardised instruments for the measurement of health literacy outcomes among Indigenous populations. It is also important for public health researchers to test skills-based health literacy measures as a focus of future research. It is ethically imperative for studies involving Indigenous Australians to openly, consistently and comprehensively collaborate with community members in all steps of the research process in order to strengthen Indigenous identity, increase self-determination and thereby enhance outcomes.

\section{Conclusion}

This review found that limited evidence exists regarding interventions to improve health literacy in Aboriginal and Torres Strait Islander Peoples. Interventions were predominantly in the form of workshops, group education or reduction of the price of healthful foods and beverages. Whilst all interventions reported improvement in at least one health literacy outcome measure, the methodological quality was weakened by small sample sizes, poor attendance, and significant loss to follow-up. It is suggested that future research should involve substantial Indigenous community engagement in all aspects of the design and implementation of interventions, including careful consideration of culture, Indigenous concepts of health and well-being, as well as language. The reciprocal sharing of ideas, promotion of respect and enhancement of participation to strengthen autonomy and build empowerment among Aboriginal and Torres Strait Islander Peoples is key to improving health literacy and in doing so, reducing disparities and a building a healthier future.

\section{Supplementary Information}

The online version contains supplementary material available at https://doi. org/10.1186/s12889-021-10278-x

Additional file 1. PRISMA checklist. 
Additional file 2. Search Strategy (Embase OVID).

Additional file 3. Reasons for exclusion of studies.

Additional file 4. JBI Critical Appraisal Checklist for Randomised Control Trials.

Additional file 5. JBI Checklist for Quasi-Experimental Studies.

\section{Abbreviations}

ABS: Australian Bureau of Statistics; AlHW: Australian Institute of Health and Welfare; ALPA: Arnhem Land Progress Aboriginal Corporation; ATSI: Aboriginal and Torres Strait Islander; COAG: Council of Australian Governments; BMI: Body Mass Index; BP: Blood Pressure; CRP: C-Reactive Protein; HbA1C : Glycated Haemoglobin; HeLD: Health Literacy in Dentistry; JBI: Joanna Briggs Institute; MeSH: Medical Subject Headings; MI: Multiple Imputations; NHMRC: National Health and Medical Research Council; OBS: Out Back Stores; PICOS: Population, Intervention, Comparator, Outcome, Study Design; REALM: Rapid Assessment of Health Literacy in Medicine; SSB: Sugar Sweetened Beverage; TOFHLA : Test of Functional Health Literacy in Adults; TSI : Torres Strait Islander; WHO : World Health Organization

\section{Acknowledgements}

We acknowledge the traditional custodians of the land on which we live and learn. The process of conducting this review has allowed us to deeply consider the disparities which continue to plague Indigenous Australians, particularly in relation to autonomy, health and wellbeing. We would like to thank Professor Aunty Kerrie Doyle (School of Medicine, Western Sydney University) for providing a candid, engaging and informative Cultural Proficiency Workshop on Indigenous Health. We also thank Ms. Katrina Chaudhary, School of Health Sciences Librarian at Western Sydney University, for her consultation services on the search strategy and Dr. Krista Cockrell, Sessional Academic, Western Sydney University for her insights into the topic. We acknowledge Ms. Samantha Togni Senior Research Officer, Menzies School of Health Research, Dr. Caroline Nilson Senior Lecturer in Nursing, Murdoch University and Associate Professor Sue Crengle, Senior Māori Health Researcher, University of Otago for providing further information on their studies and words of encouragement. Lastly, the first author (SN) would like to thank her amazing husband and family for their constant support through her public health degree.

\section{Authors' contributions}

SN led the design, search strategy, drafting and revisions of the manuscript. AA was involved in the conception and design, search strategy, providing advice, and revisions of the manuscript. Both authors read and approved the final manuscript.

\section{Funding}

AA was supported by Australian National Health and Medical Research Council Grants 1033213 and 1069861.

\section{Availability of data and materials}

The datasets generated during and/or analysed during the current study are available from the corresponding author on reasonable request.

\section{Ethics approval and consent to participate}

Not Applicable.

\section{Consent for publication}

Not Applicable.

\section{Competing interests}

AA is an Associate Editor of BMC Public Health but did not play any role in the peer-review and decision-making process for this manuscript.

\section{Author details}

${ }^{1}$ School of Health Sciences, Western Sydney University, Locked Bag 1797, Penrith, NSW 2751, Australia. ${ }^{2}$ Translational Health Research Institute, Western Sydney University, Locked Bag 1797, Penrith, NSW 2751, Australia. ${ }^{3}$ Discipline of Child and Adolescent Health, Sydney Medical School, Faculty of Medicine and Health, Westmead, NSW 2145, Australia. ${ }^{4}$ Oral Health Services, Sydney
Local Health District and Sydney Dental Hospital, Surry Hills, NSW 2010, Australia.

Received: 11 November 2019 Accepted: 19 January 2021

Published online: 30 January 2021

\section{References}

1. Nutbeam D. The evolving concept of health literacy. Soc Sci Med. 2008;67: 2072-8

2. Committee on Health Literacy, Institute of Medicine. Health Literacy: A Prescription to End Confusion. Washington DC: National Academies Press; 2004.

3. Pleasant A. Advancing health literacy measurement: a pathway to better health and health system performance. J Health Commun. 2014;19:1481-96. https://doi.org/10.1080/10810730.2014.954083.

4. Coleman C, Kurtz-Rossi S, McKinney J, Pleasant A, Rootman I, Shohet L. Calgary charter on health literacy. Montreal: The Centre for Literacy; 2009. http://www.centreforliteracy.qc.ca/sites/default/files/CFL_Calgary_Charter_2 011.pdf. Accessed 2 Nov 2020

5. Liu C, Wang D, Liu C, Jiang J, Wang $X$, Chen H, et al. What is the meaning of health literacy? A systematic review and qualitative synthesis Fam Med Community Health. 2020;8:e000351. https://doi.org/10.1136/fmch-2020000351.

6. Government of Victoria. Health literacy: Enabling communication and participation in health: Background paper. Melbourne: Department of Health; 2013.

7. Osborne RH, Batterham RW, Elsworth GR, Hawkins M, Buchbinder R. The grounded psychometric development and initial validation of the health literacy questionnaire (HLQ). BMC Public Health. 2013;13:658. https://doi.org/ 10.1186/1471-2458-13-658.

8. Edwards M, Wood F, Davies M, Edwards A. 'Distributed health literacy': longitudinal qualitative analysis of the roles of health literacy mediators and social networks of people living with a long-term health condition. Health Expect. 2015;18:1180-93. https://doi.org/10.1111/hex.12093.

9. Nutbeam D, Lloyd JE. Understanding and responding to health literacy as a social determinant of health. Annu Rev Public Health. 2021;42. https://doi. org/10.1146/annurev-publhealth-090419-102529.

10. Nutbeam D. Health literacy as a public health goal: a challenge for contemporary health education and communication strategies into the $21 \mathrm{st}$ century. Health Promot Int. 2000;15:259-67.

11. World Health Organization. WHO health evidence network synthesis report 65: what is the evidence on the methods, frameworks and indicators used to evaluate health literacy policies, programmes and interventions at the regional, national and organizational levels? Geneva: World Health Organization; 2019.

12. Berkman ND, Sheridan SL, Donahue KE, Halpern DJ, Crotty K. Low health literacy and health outcomes: an updated systematic review. Ann Intern Med. 2011;155:97-107.

13. Cavaleri R, Mapedzahama V, Firdaus R, Ayika D, Arora A. Culturally and linguistically diverse Australians. In: Dune T, McLeod K, Williams R, editors. Culture, diversity and health in Australia: towards culturally safe health care. UK: Routledge; 2021.

14. Australian Bureau of Statistics. Census of Population and Housing: Reflecting Australia - Stories from the Census, 2016. Canberra: Australian bureau of statistics; 2017 https://www.absgovau/ausstats/abs@nsf/Lookup/by\%2 OSubject/20710 2016 Main\%20Features Cultural\%20Diversity\%20Data\%2 OSummary 30\#: :text=Over\%20300\%20ancestries\%20were\%2 Oseparately,\%25)\%20and\%20Indian\%20(46\%25). Accessed 1 Aug 2020.

15. Australian Bureau of Statistics. Estimates of Aboriginal and Torres Strait Islander Australians, June 2016. Canberra: Australian bureau of statistics; 2018 https://wwwabsgovau/ausstats/abs@nsf/mf/3238055001 Accessed 1 Aug 2020.

16. Leske S, Harris MG, Charlson FJ, Ferrari AJ, Baxter AJ, Logan JM, et al. Systematic review of interventions for indigenous adults with mental and substance use disorders in Australia, Canada, New Zealand and the United States. Aust N Z J Psychiatry. 2016;50:1040-54.

17. Australian Institute of Health and Welfare. The health and welfare of Australia's Aboriginal and Torres Strait islander peoples. Canberra: Australian Institute of Health and Welfare; 2015.

18. Marmot M. Social determinants and the health of indigenous Australians. Med J Aust. 2011;194:512-3. 
19. Australian Government Department of Prime Minister and Cabinet. Closing the gap report 2019. Canberra; 2019. https://trove.nla.gov.au/work/2351 99245? selectedversion=NBD65007437. Accessed 3 Nov 2019.

20. Alford KA. Indigenous health expenditure deficits obscured in closing the gap reports. Medi J Aust. 2015;203:403.

21. Department of Health and Aging. National Aboriginal and Torres Strait islander health plan 2013-2023. Canberra: Department of Health and Aging; 2013.

22. King M, Smith A, Gracey M. Indigenous health part 2: the underlying causes of the health gap. Lancet. 2009;374:76-85.

23. Australian Government Department of Health and Ageing. National Aboriginal and Torres Strait Islander Health Plan 2013-2023. Canberra: Australian Government Department of Health and Ageing; 2013.

24. Australian Commission on Safety and Quality in Health Care. Health literacy: Taking action to improve safety and quality. Sydney: Australian Commission on Safety and Quality in Health Care; 2014. https://www.safetyandquality. gov.au/sites/default/files/migrated/Health-Literacy-Taking-action-to-improvesafety-and-quality.pdf Accessed 11 Nov 2020.

25. Australian Institute of Health and Welfare. Closing the Gap targets: 2017 analysis of progress and key drivers of change. Canberra: Australian Institute of Health and Welfare; 2018.

26. Commonwealth of Australia, Department of the Prime Minister and the Cabinet. Closing the Gap Report 2020. Canberra: Commonwealth of Australia. https://ctgreport.niaa.gov.au/sites/default/files/pdf/closing-the-gapreport-2020.pdf (accessed 1 Aug 2020).

27. Rheault $H$, Coyer $F$, Jones $L$, Bonner A. Health literacy in indigenous people with chronic disease living in remote Australia. BMC Health Serv Res. 2019; 19:523. https://doi.org/10.1186/s12913-019-4335-3.

28. Australian Bureau of Statistics. Australian Social Trends: Health Literacy 2006. Canberra; 2008. http://www.ausstats.abs.gov.au/Ausstats/subscriber.nsf/0/73 ED158C6B14BB5ECA2574720011AB83/\$File/42330_2006.pdf. (accessed 22 Aug 2019).

29. Australian Bureau of Statistics. National Health Survey: Health Literacy, 2018. Canberra; 2019. https://www.abs.gov.au/ausstats/abs@.nsf/mf/4364.0.55.014 (accessed 1 Aug 2020).

30. Sheridan SL, Halpern DJ, Viera AJ, Berkman ND, Donahue KE, Crotty K. Interventions for individuals with low health literacy: a systematic review. J Health Commun. 2011;16(Suppl 3):30-54.

31. Taggart J, Williams A, Dennis S, Newall A, Shortus T, Zwar N, et al. A systematic review of interventions in primary care to improve health literacy for chronic disease behavioural risk factors. BMC Fam Pract. 2012:13:49.

32. Thewes B, McCaffery K, Davis E, Garvey G. Insufficient evidence on health literacy amongst indigenous people with cancer: a systematic literature review. Health Promot Int. 2018;33:195-218.

33. Choudhry FR, Ming LC, Munawar K, Zaidi STR, Patel RP, Khan TM, et al. Health literacy studies conducted in Australia: a scoping review. Int J Environ Res Public Health. 2019;16:1112.

34. Clifford A, McCalman J, Bainbridge R, Tsey K. Interventions to improve cultural competency in health care for indigenous peoples of Australia, New Zealand, Canada and the USA: a systematic review. Int J Qual Health Care. 2015;27:89-98

35. Clifford AC, Doran CM, Tsey K. A systematic review of suicide prevention interventions targeting indigenous peoples in Australiam United States. Canada and New Zealand BMC Public Health. 2013;13:11.

36. Moher D, Liberati A, Tetzlaff J, Altman DG. The Prisma group. Preferred Reporting Items for Systematic Reviews and Meta-Analyses: The PRISMA Statement. PLOS Med. 2009;6:e1000097.

37. Booth A, Clarke M, Dooley G, Ghersi D, Moher D, Petticrew M, et al. The nuts and bolts of PROSPERO: an international prospective register of systematic reviews. Syst Rev. 2012;1:2. https://doi.org/10.1186/2046-4053-1-2.

38. Costantino G, Montano N, Casazza G. When should we change our clinical practice based on the results of a clinical study? Searching for evidence: PICOS and PubMed. Intern Emerg Med. 2015;10:525-7.

39. Analytics C. EndNote X9.1.1. San Francisco: Clarivate Analytics; 2019.

40. Higgins JPT, Deeks JJ. Selecting studies and collecting data. In: Higgins JPT, Green S, editors. Cochrane handbook for systematic reviews of interventions. New Jersey: Wiley; 2008. p. 151-85.

41. Brimblecombe J, Ferguson M, Barzi F, Brown C, Ball K. Mediators and moderators of nutrition intervention effects in remote indigenous Australia. Br J Nutr. 2018;119:1424-33.
42. Brimblecombe J, Ferguson M, Chatfield MD, Liberato SC, Gunther A, Ball K, et al. Effect of a price discount and consumer education strategy on food and beverage purchases in remote indigenous Australia: a stepped-wedge randomised controlled trial. Lancet Public Health. 2017:2:e82-95.

43. Canuto K, Cargo M, Li M, D'Onise K, Esterman A, McDermott R. Pragmatic randomised trial of a 12-week exercise and nutrition program for Aboriginal and Torres Strait islander women: clinical results immediate post and 3 months follow-up. BMC Public Health. 2012;12:933.

44. Ju XQ, Brennan D, Parker E, Mills H, Kapellas K, Jamieson L. Efficacy of an oral health literacy intervention among indigenous Australian adults. Community Dent Oral Epidemiol. 2017:45:413-26.

45. Mills K, Gatton ML, Mahoney R, Nelson A. 'Work it out': evaluation of a chronic condition self-management program for urban Aboriginal and Torres Strait islander people, with or at risk of cardiovascular disease. BMC Health Serv Res. 2017;17:680.

46. Pettigrew S, Jongenelis MI, Moore S, Pratt IS. A comparison of the effectiveness of an adult nutrition education program for Aboriginal and non-Aboriginal Australians. Soc Sci Med. 2015;145:120-4.

47. Tufanaru C, Munn Z, Aromataris E, Campbell J, Hopp L. Systematic reviews of effectiveness. In: Aromataris E, Munn Z (editors). Joanna Briggs Institute Reviewer's Manual. Adelaide: Joanna Briggs Institute; 2017. https:// reviewersmanual.joannabriggs.org/. Accessed 12 May 2019.

48. Walker M, Fredericks B, Mills K, Anderson D. 'Yarning' as a method for community-based health research with indigenous women: the indigenous women's wellness research program. Health Care for Women Int. 2014;35: $1216-26$.

49. Institute of Medicine. Measures of health literacy: workshop summary. Washington DC: The National Academies Press; 2009.

50. Australian Commission on Safety and Quality in Healthcare. Health Literacy: Taking action to improve safety and quality Sydney: Australian Commission on Safety and Quality in Healthcare; 2014

51. Rubin DL, Freimuth VS, Johnson SD, Kaley T, Parmer J. Training meals on wheels volunteers as health literacy coaches for older adults. Health Promot Prac. 2014;15:448-54.

52. Prescott GM, Dascanio SA, Klosko R, Shogan M. Development of a medication health literacy program for refugees. J Am Pharm Assoc. 2018:58:673-8.

53. Lee $\mathrm{H}, \mathrm{Ho}$ PS, Wang WC, Hu CY, Lee $\mathrm{CH}$, Huang HL. Effectiveness of a health belief model intervention using a lay health advisor strategy on mouth selfexamination and cancer screening in remote aboriginal communities: A randomized controlled trial. Patient Educ Couns. 2019. https://doi.org/10. 1016/j.pec.2019.07.001.

54. Crengle S, Luke JN, Lambert M, Smylie JK, Reid S, Harre-Hindmarsh J, et al. Effect of a health literacy intervention trial on knowledge about cardiovascular disease medications among indigenous peoples in Australia. Canada and New Zealand BMJ Open. 2018:8:e018569.

55. Slade GD, Bailie RS, Roberts-Thomson K, Leach AJ, Raye I, Endean C, et al. Effect of health promotion and fluoride varnish on dental caries among Australian Aboriginal children: results from a community-randomized controlled trial. Community Dent Oral Epidemiol. 2011:39:29-43.

56. Valery PC, Masters IB, Taylor B, Laifoo Y, O'Rourke PK, Chang AB. An education intervention for childhood asthma by Aboriginal and Torres Strait islander health workers: a randomised controlled trial. Med J Aust. 2010;192:574-9.

57. Ni Mhurchu C, Blakely T, Jiang Y, Eyles HC, Rodgers A. Effects of price discounts and tailored nutrition education on supermarket purchases: a randomised control trial. Am J Clin Nutr. 2010;91:736-47.

58. Waterlander WE, de Boer MR, Schuit AJ, Seidell JC, Steenhuis IH. Price discounts significantly enhance fruit and vegetable purchases when combined with nutrition education: a randomised controlled supermarket trial. Am J Clin Nutr. 2013;97:886-95.

59. Ferguson M, O'Dea K, Holden S, Miles E, Brimblecombe J. Food and beverage discounts to improve health in remote Aboriginal communities: mixed method evaluation of a natural experiment. Aust NZ J Public Health. 2017:41:32-7.

60. World Cancer Research Fund International. Building momentum: lessons on implementing a robust sugar sweetened beverage tax 2018. London: World Cancer Research Fund International; 2018.

61. Obesity Policy Coalition. The case for a health levy on sugar-sweetened beverages: Policy brief. Melbourne: Obesity Policy Coalition; 2018.

62. Becker NV, Asch DA, Kullgreen JT, Bellamy SL, Sen AP, Volpp KG. Stages of change and patient activation measure scores in the context of incentivebased health interventions. Amer J Health Promot. 2015;30:133-5. 
63. Zutlevics TL. Could providing financial incentives to research participants be ultimately self-defeating? Res Ethics. 2016;12:137-48.

64. Malseed C, Nelson A, Ware R, Lacey I, Lander K. Deadly choices community health events: a health promotion initiative for urban Aboriginal and Torres Strait islander people. Aust J Prim Health. 2014;20:379-83.

65. Biggs K, Walsh J, Ooi C. Deadly liver mob: opening the door - improving sexual health pathways for Aboriginal people in Western Sydney. Sex Health. 2016;13:457-64.

66. Gould GS, Bovill M, Pollock L, Bonevski B, Gruppetta M, Atkins L, et al. Feasibility and acceptability of indigenous Counselling and nicotine (ICAN) QUIT in pregnancy multicomponent implementation intervention and study design for Australian indigenous pregnant women: a pilot cluster randomised step-wedge trial. Addict Behav. 2019;90:176-90.

67. Higgins ST, Silverman K, Sigmon SC, Naito NA. Incentives and health: an introduction. Prev Med. 2012:55:52-6.

68. Jessup RL, Osborne RH, Buchbinder R, Beauchamp A. Using co-design to develop interventions to address health literacy needs in a hospitalised population. BMC Health Serv Res. 2018;18:989.

69. Chamberlain C, Gee G, Brown SJ, Atkinson J, Herrman H, Gartland D, et al. Healing the past by nurturing the future - co-designing perinatal strategies for Aboriginal and Torres Strait islander parents experiencing complex trauma: framework and protocol for a community-based participatory action research study. BMJ Open. 2019;9:e028397.

70. Australian Government. Our People: Indigenous peoples and cultures. Canberra; 2019 https://www.australia.gov.au/about-australia/our-country/ our-people. Accessed 27 Oct 2019.

71. Vass A, Mitchell A, Dhurrkay Y. Health literacy and Australian indigenous peoples: an analysis of the role of language and worldview. Health Promotion Journal of Australia. 2011;22:33-7.

72. Togni S. The Uti Kulintjaku project: the path to clear thinking. An evaluation of an innovative, Aboriginal-led approach to developing bi-cultural understanding of mental health and wellbeing. Aust Psychol. 2016;52(4): 268-79.

\section{Publisher's Note}

Springer Nature remains neutral with regard to jurisdictional claims in published maps and institutional affiliations.

Ready to submit your research? Choose BMC and benefit from:

- fast, convenient online submission

- thorough peer review by experienced researchers in your field

- rapid publication on acceptance

- support for research data, including large and complex data types

- gold Open Access which fosters wider collaboration and increased citations

- maximum visibility for your research: over $100 \mathrm{M}$ website views per year

At $\mathrm{BMC}$, research is always in progress.

Learn more biomedcentral.com/submissions 